\title{
¿Es Alstroemeria pulchra Sims var. maxima Phil. (Alstroemeriaceae) un miembro del complejo A. magnifica Herbert?: Estudio colorimétrico de los tépalos externos e internos de la flor*
}

\section{Is Alstroemeria pulchra Sims var. maxima Phil. (Alstroemeriaceae) a member of the $A$. magnifica Herbert complex?: A colorimetric study of outer and inner flower tepals*}

\author{
Víctor L. Finot ${ }^{1 *}$, Carlos M. Baeza ${ }^{2}$, Pedro Melín ${ }^{4}$, Patricio Novoa ${ }^{3}$, Jorge Campos ${ }^{1}$, Eduardo \\ Ruiz $^{2}$, Glenda Fuentes ${ }^{2}$, Paula Albornoz $z^{4}$ Pedro Carrasco ${ }^{2}$ \\ 1'Departamento de Producción Animal, Facultad de Agronomía, Universidad de Concepción, Casilla 537, Chillán, \\ Chile. \\ ${ }^{2}$ Departamento de Botánica, Facultad de Ciencias Naturales y Oceanográficas, Casilla 160-C, Universidad de \\ Concepción, Concepción, Chile. \\ ${ }^{3}$ Jardín Botánico Nacional, Viña del Mar, Chile. \\ ${ }^{4}$ Facultad de Ingeniería Agrícola, Universidad de Concepción, Casilla 537, Chillán, Chile. \\ **vifinot@udec.cl
}

\begin{abstract}
RESUMEN
Alstroemeria pulchra var. maxima es un taxón endémico de Chile descrito por R.A. Philippi en 1864, cuya posición sistemática es controversial; aunque Bayer en 1987 transfiere este taxón a A. magnifica con el rango de subespecie, Muñoz \& Moreira en 2003 retoman el concepto original de Philippi. Con el objetivo de proporcionar nuevos caracteres para decidir si A. pulchra var. maxima es un miembro del complejo A. magnifica o debe permanecer como una variedad de A. pulchra como fue originalmente descrita, se analizó el color de los tépalos externos, internos superiores e interno inferior, utilizando un espectrofotómetro colorímetro Hunterlab Colorquest. Se trabajó con flores de ocho poblaciones de Alstroemeria L. pertenecientes a estas dos especies endémicas de Chile: cinco poblaciones de A. magnifica Herbert y tres poblaciones de A. pulchra Sims. Las mediciones en el espacio CIELab permiten distinguir A. magnifica de A. pulchra sobre la base del color de los tépalos externos y del tépalo interno inferior, pero no por el color de los tépalos internos superiores. Se concluye que el color de la flor es un nuevo carácter que confirma que la var. maxima pertenece a A. pulchra y no al complejo A. magnifica.
\end{abstract}

Palabras clave: CIELab, color de la flor, especies endémicas, Flora de Chile, tépalos.

\begin{abstract}
Alstroemeria pulchra var. maxima is an endemic Chilean taxon described by R.A. Philippi in 1864, whose taxonomic position is controversial; even though Bayer in 1987 transferred this taxon to A. magnifica with the rank of subspecies, Muñoz \& Moreira in 2003 return to the original concept proposed by Philippi. In order to provide a new character to decide if $A$. pulchra var. maxima is a member of the A. magnifica complex or must remain as a variety of A. pulchra as was originally described, the color of outer, upper inner and lower inner tepals of the flowers of eight populations of Alstroemeria L. belonging to these two endemic Chilean species were analyzed with a spectrophotometer colorimeter Hunterlab Colorquest: five populations of $A$. magnifica Herbert and three populations of $A$. pulchra Sims. CIELab coordinates allow to separate A. magnifica from A. pulchra on the basis of the color of the outer and lower inner tepals but not by the color of the upper inner tepals. We conclude that the color of the flower is a new character to confirm that var. maxima belong to $A$. pulchra, not to the $A$. magnifica complex.
\end{abstract}

KEYwoRDs: CIELab, color of flowers, endemic species, Flora of Chile, tepals.

\section{INTRODUCCIÓN}

El género Alstroemeria L. (Alstroemeriaceae) es endémico de Sudamérica. Comprende unos 82 taxones desde Venezuela a Tierra del Fuego, con dos centros de distribución: Chile y
Brasil (Uphof 1952, Bayer 1987, Han et al. 2000, Muñoz \& Moreira 2003, Ruiz et al. 2010, Chacón et al. 2012). En Chile existen unos 49 taxones distribuidos a lo largo de todo el país $\left(20-53^{\circ} \mathrm{S}\right)$, aunque la mayor concentración de especies ocurre entre los $28^{\circ} \mathrm{S}$ y $37^{\circ} \mathrm{S}$ (Muñoz \& Moreira 2003, Ruiz et al.

* Dedicamos este trabajo a la memoria del Prof. Clodomiro Marticorena Pairoa. 
2010). Unos 42 taxones, esto es más del $80 \%$ de los taxones chilenos, son endémicos, la mayor parte con distribución geográfica muy restringida (Muñoz \& Moreira 2003).

Las especies de Alstroemeria son hierbas perennes, rizomatozas, apreciadas por su valor ornamental (Rustanius et al. 1991, Buitendijk \& Ramanna 1996, Buitendijk et al. 1997, Kashihara et al. 2011, Baeza et al. 2010, Ruiz et al. 2010). Varias especies chilenas son parentales de híbridos interespecíficos utilizados para el cultivo, entre las cuales podemos mencionar $A$. aurea Graham, A. pelegrina L., A. ligtu L., A. pulchra Sims y A. magnifica Herbet, entre otras (Ruiz et al. 2010).

La clasificación de las especies se basa en características de la inflorescencia, flor, tallo, hojas, frutos y rizomas (Bayer 1987, Han et al. 2000, Muñoz \& Moreira 2003). Entre las características florales de valor taxonómico previamente consideradas se encuentran el tamaño de las piezas florales, el color de la base de los tépalos internos superiores y sus patrones de dibujos (máculas), la disposición de los tépalos externos, el ápice de los tépalos externos y el tamaño de los tépalos internos superiores respecto de los demás (Muñoz \& Moreira 2003). Las flores de Alstroemeria son generalmente zigomorfas (muy raramente actinomorfas), trímeras y epíginas. Poseen un perigonio formado por 6 tépalos; los 3 tépalos externos son iguales entre sí tanto en tamaño como en color, mientras los tépalos internos superiores se distinguen del tépalo interno inferior por la forma, tamaño y color. Los tépalos internos superiores son más largos y angostos que el inferior, poseen complejos patrones de manchas, a menudo, sobre más de un color de fondo y poseen nectarios en la base; el tépalo interno inferior suele ser similar, en forma y color, a los tépalos externos (Sanso 1996). Caracteres anatómicos tienen también potencial valor taxonómico (Lyshede 2002).

Alstroemeria pulchra var. maxima Phil. es un taxón endémico de Chile descrito por R.A. Philippi en 1864, sobre la base de un espécimen que él había recolectado en 1860 en Cajón del Boldo, Aconcagua, Región de Valparaíso. El nombre varietal alude a su tamaño que puede superar 1,70 $\mathrm{m}$ de alto, con inflorescencias de hasta $20 \mathrm{~cm}$ de diámetro y grandes flores de ca. $10 \mathrm{~cm}$ de diámetro, de color lila (Fig. 1A-C). Muñoz \& Moreira (2003) establecen su distribución entre el Cerro Pan de Azúcar (2959’ S, Región de Coquimbo) y Cuesta Lo Prado (3328' S, Región Metropolitana).

Bayer (1987) considera que esta variedad es más cercana a $A$. magnifica Herbert que a $A$. pulchra y la transfiere con el rango de subespecie [A. magnifica subsp. maxima (Phil.) Bayer]. Posteriormente, Muñoz \& Moreira (2003) en su libro sobre las Alstroemerias de Chile retoman el concepto original propuesto por Philippi (1864) para este taxón. Estos autores sostienen que el largo relativo de los tépalos internos superiores más largos y que sobresalen notoriamente de los demás, la hacen más afín a $A$. pulchra.

Alstroemeria magnifica es una especie endémica de Chile muy frecuente entre las regiones de Coquimbo y Valparaíso, perteneciente al clado chileno-central (Aaegesen $\&$ Sanso 2003). Se caracteriza por sus flores de color lila o violáceo pálidas en los tépalos externos e interno inferior, mientras los tépalos internos superiores manifiestan un color blanco en la base, amarillo en la mitad superior y líneas de color púrpura, con una gran mancha purpúrea tanto en el ápice como en la base. Las flores de $A$. magnifica son especialmente hermosas y es una de las especies chilenas con flores más grandes. Su potencial como especie ornamental es enorme, como lo es en general para todo el género Alstroemeria (Kashihara et al. 2011, Assis 2004). De esta especie se han descrito varios taxones infraespecíficos: Alstroemeria magnifica var. magnifica, A. magnifica var. sierrae (Muñoz) Muñoz-Schick y A. magnifica var. tofoensis Muñoz-Schick, restringidas a la Región de Coquimbo, desde el litoral costero hasta los cerros de la Cordillera de la Costa (Muñoz \& Moreira 2003) y Alstroemeria magnifica var. magenta (Ehr. Bayer) Muñoz-Schick, endémica de las regiones de Coquimbo y Valparaíso.

Por su valor ornamental, el estudio del color de las flores de Alstroemeria ha concitado la atención de varios autores (Saito et al. 1985, 1988, Norbaek et al. 1996, 1998, Kashihara et al. 2011). En el género Alstroemeria es posible encontrar flores blancas, amarillas, rosadas, anaranjadas, rojas y purpúreas (Bayer 1987); los tres últimos colores son producidos por antocianinas. En Alstroemeria se ha demostrado la existencia de 7 antocianinas (6-hidroxidelfinidina 3-rutinósido, 6-hidroxicianidina 3-rutinósido, cianidina 3-rutinósido, delfinidina 3-rutinósido, cianidina 3-malonilglucósido, 6-hidroxicianidina 3-malonilrutinósido y delfinidina 3-malonilglucósido), que la intensidad del color se relaciona positivamente con la concentración de estos pigmentos y que la co-pigmentación con flavonoides causa un cambio en los tonos azules, independiente de los pigmentos antociánicos (Norbaek et al. 1996, 1998). Según Norbaek et al. (1996), las antocianinas más abundantes en $A$. pulchra var. maxima Phil. [y en A. magnifica Herbert subsp. maxima (Phil.) Bayer, tratada como un taxón diferente], son cianidina 3-rutinósido y cianidina 3-malonilglucósido, carácter que comparte con A. magenta Bayer (= A. magnifica var. magenta). Norbaek et al. (1996) otorgan valor taxonómico a la presencia de cianidina 3-malonilglucósido, dado que sólo 18 de las 28 especies estudiadas tienen la capacidad de producir estos pigmentos.

Aplicaciones taxonómicas de los análisis colorimétricos de las flores de Alstroemeria son virtualmente inexistentes, aunque han sido aplicados con este fin en otros grupos taxonómicos, como Paeonia (Wang et al. 2004), Solanum (Fajardo et al. 2008) y Gerbera (Hatamzadeh et al. 2012). No obstante, se ha sugerido que las antocianinas pueden ser utilizadas con propósitos taxonómicos en Alstroemeria (Norbaek et al. 1996). En el presente trabajo se analizó el color de los tépalos externos, internos superiores e interno inferior de A. magnifica, A. pulchra var. pulchra y A. pulchra var. maxima con el propósito de buscar nuevos caracteres que permitan aclarar la posición taxonómica de $A$. pulchra var. maxima. 

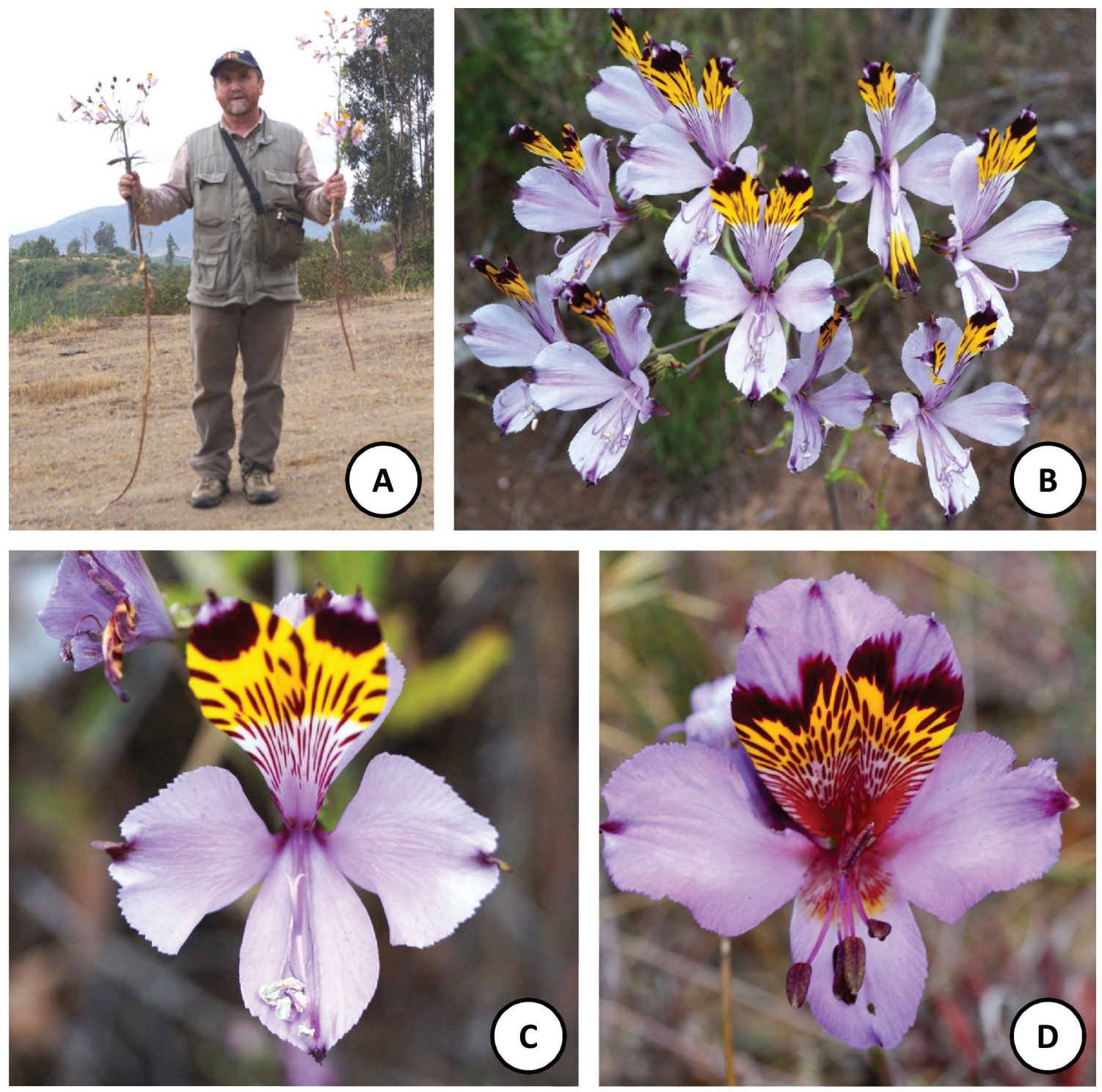

Figura 1. Aspecto general de la inflorescencia y flor en el complejo Alstroemeria magnifica. A-C. Alstroemeria pulchra var. maxima. A. Uno de los autores (CMB) mostrando dos plantas recolectadas en Cuesta El Melón el 30 de noviembre de 2013. B. Inflorescencia. C. Flor. D. Alstroemeria magnifica. Flor fotografiada en bosque Santa Julia, Los Vilos. A-C de Patricio Novoa. D de Víctor Finot.

FIGURE 1. General aspect of the flower and inflorescence in the Alstroemeria magnifica complex. A-C. Alstroemeria pulchra var. maxima. A. One of the authors (CMB) showing two plants collected at Cuesta El Melón on November 30, 2013. B. Inflorescence. C. Flower. D. Alstroemeria magnifica. Flower photographed in Santa Julia wood, Los Vilos. A-C by Patricio Novoa. D by Victor L. Finot.

\section{MATERIALES Y MÉTODOS}

RECOLECCIÓN DE LAS FLORES

Flores de Alstroemeria magnifica y A. pulchra fueron recolectadas entre el 30 de octubre y el 1 de noviembre de 2013, en las regiones de Coquimbo y Valparaíso (Tabla I). Se estudiaron 8 poblaciones, 5 de $A$. magnifica y 3 poblaciones de $A$. pulchra. Las flores fueron conservadas húmedas en frío $\mathrm{y}$ transportadas al laboratorio para análisis colorimétrico.

\section{ANÁLISIS COLORIMÉTRICO}

Para el análisis de color, los tépalos de cada flor (10 flores por población, total 80 flores) fueron separados en tépalos externos $(\mathrm{n}=240)$, internos superiores $(\mathrm{n}=160)$, e interno inferior $(\mathrm{n}=$ 80); el análisis se realizó en la zona media de la cara adaxial de tépalos frescos, utilizando al menos 5 flores por población, tomadas de distintos individuos. Se midió luminosidad ( $\left.\mathrm{L}^{*}\right)$ y los parámetros $\mathrm{a}^{*} \mathrm{y} \mathrm{b}^{*}$ de las coordenadas de color del espacio CIELab (Commission Internationale de l'Eclairage, 1978) en un espectrofotómetro colorímetro Hunterlab Colorquest; se 
utilizó el promedio de 3 mediciones sucesivas. L* representa la claridad o luminosidad relativa de los colores en una escala de 0 a 100, donde valores bajos indican oscuro y valores altos indican luz en sucesiva escala de grises hasta llegar al color blanco (100); los valores positivos de a* indican rojo y los valores negativos indican verde en tanto el parámetro $b^{*}$ tiene valores negativos para azul y positivos para amarillo. El valor $\mathrm{C}^{*}$ (croma o saturación) se calculó como $\left(\mathrm{a}^{* 2}+\mathrm{b}^{* 2}\right)^{1 / 2}$ y el valor $\mathrm{h}$ (tono, hue) fue calculado como $\operatorname{arctg}\left(\mathrm{b}^{*} / \mathrm{a}^{*}\right)$ (Sacks \& Shaw 1994, Wang et al. 2001, 2004). Croma representa la intensidad de un color, de modo que valores bajos indican opacidad (más cercano al gris) y valores altos representan brillantez (más cercanos al color puro). El valor de tono o matiz (h) representa una posición en la escala cromática. La diferencia total en color entre los taxones estudiados $(\Delta \mathrm{E})$ se calculó por la expresión: $\Delta \mathrm{E}^{*}=\left[\left(\Delta \mathrm{L}^{*}\right)^{2}+\left(\Delta \mathrm{a}^{*}\right)^{2}+\left(\Delta \mathrm{b}^{*}\right)^{2}\right]^{1 / 2}$ (Bañón et al. 2002).

AnÁlisis ESTAdístico

Para determinar si la posición de las poblaciones estudiadas en el espacio CIElab determinado por los parámetros $a^{*} \mathrm{y}^{*}$ (Fig. 2) es significativamente distinta se utilizó el test no paramétrico de Wilcoxson para $\mathrm{P}<0,05$. La posición de los grupos a lo largo de los ejes $\mathrm{a}^{*} \mathrm{y} \mathrm{b}^{*}$ se representó como gráficos de caja y bigote (Fig. 2). Los datos colorimétricos fueron sometidos a análisis de componentes principales. La matriz de datos comprendió 8 OTUs correspondientes a las poblaciones incluidas en el estudio y 15 variables $\left(a^{*}, b^{*}, L^{*}, C^{*}, h\right.$ por tépalos externos,

TABLA I. Taxones estudiados, localidades y fechas de recolección.

TABLE I. Studied taxa, localities and collection dates.

\begin{tabular}{|c|c|c|c|}
\hline TAXÓN & RO DE RECOLECCIÓN** & LOCALIDAD & FECHA \\
\hline A. magnifica & 4379 & $\begin{array}{l}\text { IV Región. Provincia de Choapa. Comuna de Los Vilos. Bosque Santa } \\
\text { Julia, fundo Agua Amarilla, } 31^{\circ} 49^{\prime} \text { S, } 71^{\circ} 30^{\prime} \text { W, } 110 \text { m. }\end{array}$ & $31 / 10 / 13$ \\
\hline A. magnifica & 4380 & $\begin{array}{l}\text { IV Región. Provincia de Choapa. Comuna de Los Vilos, entre quebrada } \\
\text { El Negro y Los Vilos, } 31^{\circ} 57^{\prime} \mathrm{S}, 71^{\circ} 29^{\prime} \mathrm{W}, 138 \mathrm{~m} .\end{array}$ & $31 / 10 / 13$ \\
\hline A. magnifica & 4381 & $\begin{array}{l}\text { IV Región. Provincia de Choapa. Fundo Palo Colorado, } 5 \mathrm{~km} \text { al norte } \\
\text { de Puente Quilimarí, frente al Cerro Tentén, } 32^{\circ} 05^{\prime} \mathrm{S}, 71^{\circ} 30^{\prime} \mathrm{W}, 80 \mathrm{~m} \text {. }\end{array}$ & $1 / 11 / 13$ \\
\hline A. magnifica & 4383 & $\begin{array}{l}\text { V Región. Provincia de Petorca. Comuna La Ligua. } 2 \mathrm{~km} \text { al sur de } \\
\text { Los Molles, } 32^{\circ} 14^{\prime} \mathrm{S}, 71^{\circ} 29^{\prime} \mathrm{W}, 37 \mathrm{~m} .\end{array}$ & $1 / 11 / 13$ \\
\hline A. magnifica & 4375 & $\begin{array}{l}\text { IV Región. Provincia de Coquimbo. Caleta Hornos, } 29^{\circ} 37^{\prime} \text { S, } \\
71^{\circ} 17^{\prime} \mathrm{W}, 41 \mathrm{~m} .\end{array}$ & $30 / 10 / 13$ \\
\hline A. pulchra var. maxima & 4385 & 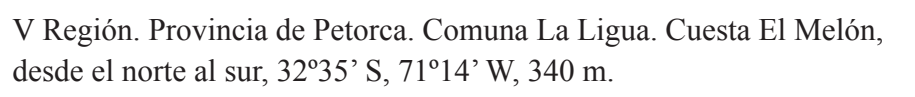 & $1 / 11 / 13$ \\
\hline A. pulchra var. maxima & 4382 & $\begin{array}{l}\text { V Región. Provincia de Petorca. Los Molles, en sitios eriazos de la } \\
\text { calle Gabriela Mistral } 1438 \text { y aledaño, } 32^{\circ} 14^{\prime} \text { S, } 71^{\circ} 31^{\prime} \text { W, } 35 \text { m. }\end{array}$ & $1 / 11 / 13$ \\
\hline A. pulchra var. pulchra & 4384 & $\begin{array}{l}\text { V Región. Provincia de Petorca. Comuna La Ligua. Los Hornos, } \\
\text { Huequén al interior, } 32^{\circ} 14^{\prime} \mathrm{S}, 71^{\circ} 20^{\prime} \mathrm{W}, 206 \mathrm{~m} \text {. }\end{array}$ & $1 / 11 / 13$ \\
\hline
\end{tabular}

internos superiores e internos inferiores) (Tabla II). El análisis de componentes principales se realizó sobre la base de datos estandarizados y se calculó un coeficiente de correlación cofenética (rcc); la posición de los grupos a lo largo de los dos primeros componentes se representó en gráficos de caja y bigote. Para determinar si la posición de los grupos delimitados a lo largo de los dos primeros componentes es significativamente distinta se utilizó el test no paramétrico de Wilcoxson para $\mathrm{P}<0,05$. Se realizó análisis de regresión lineal simple entre los parámetros CIELab; se entrega el coeficiente de regresión ajustado (raj2) y la curva de regresión (Fig. 3). La variabilidad de los caracteres colorimétricos en A. magnifica y A. pulchra se analizó mediante gráficos de caja y bigote. Todos los análisis se realizaron utilizando el programa Infostat v. 2013 (Di Rienzo et al. 2013).

\section{RESULTADOS}

Los valores de los parámetros $\mathrm{a}^{*}, \mathrm{~b}^{*}$, luminosidad $\left(\mathrm{L}^{*}\right)$, croma (C*) y tono (h) se muestran en la Tabla II. Los espectros de reflectancia de los tépalos externos, internos superiores e internos inferiores de las ocho poblaciones estudiadas se muestran en la Figura 4. La distribución de las poblaciones analizadas en el espacio determinado por los parámetros a* (verde/rojo), b* (azul/ amarillo) se muestra en la Figura 2. Las diferencias en el color de los tépalos entre los taxones estudiados se muestran en la Tabla III. La variabilidad de los caracteres colorimétricos entre $A$. pulchra y A. magnifica se muestra en la Figura 5. Los valores de $a^{*}$ de los tépalos externos, internos superiores e interno inferior fueron siempre positivos, indicando predominancia del color rojo. Los valores de $b^{*}$ de tépalos externos e internos inferiores 

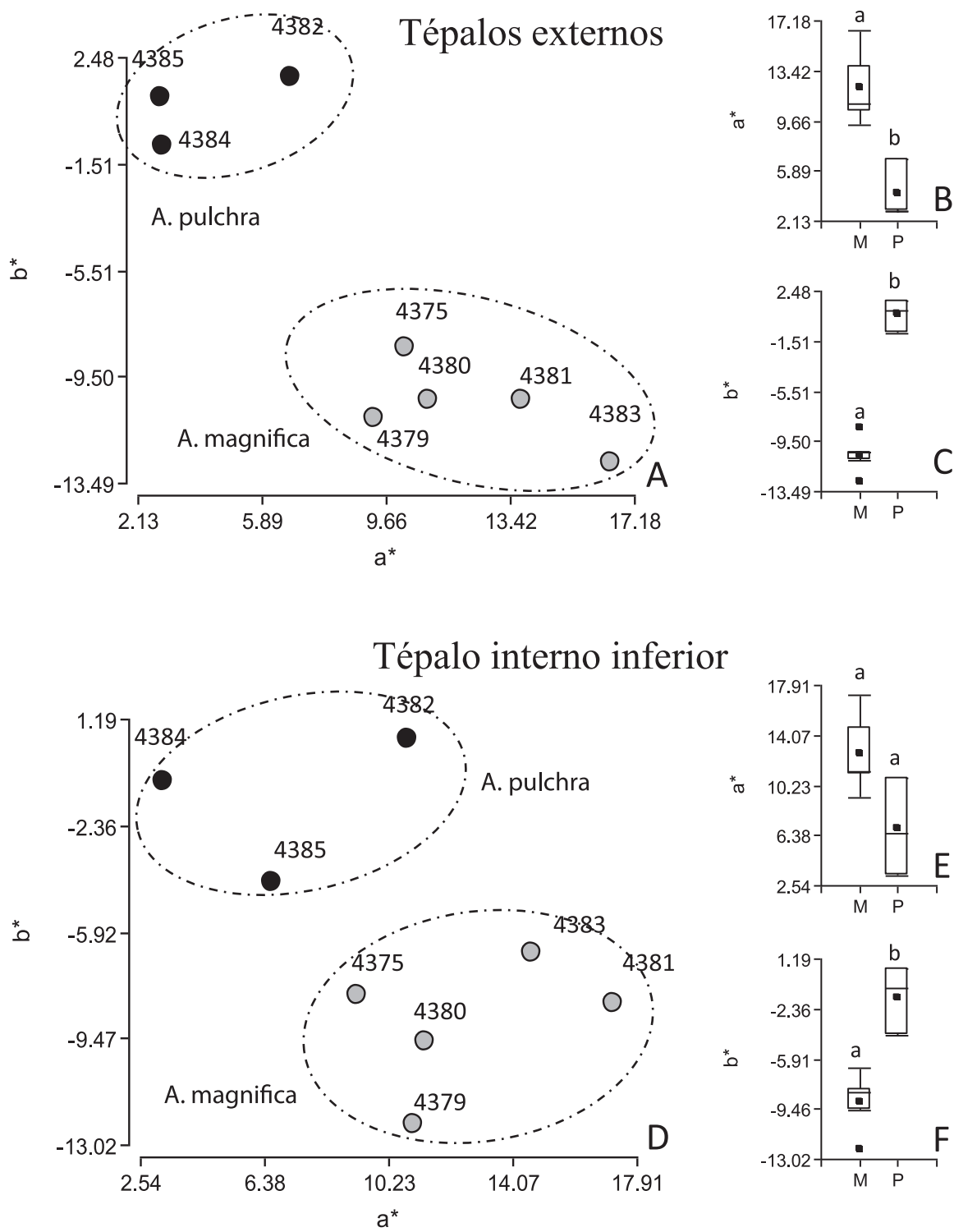

Figura 2. Distribución de los taxones estudiados en el espacio CIELab determinado por las coordenadas a* y b*. A-C. Tépalos externos. D-F. Tépalo interno inferior. Los rótulos corresponden a las poblaciones como se indica en la Tabla I. $\bigcirc=A$. magnifica; $\mathbf{O}=A$. pulchra .

Figure 2. Distribution of the studied taxa in the CIELab space determined by the coordinates a* and $\mathrm{b}^{*}$. A-C. Outer tepals. D-F. Lower inner tepal. Labels correspond to populations as indicated in Table I. $\mathrm{O}=$ A. magnifica; $\mathbf{O}=$ A. pulchra.

fueron negativos o cercanos a cero, indicando predominancia del color azul. Los tépalos internos superiores presentaron, en cambio, valores de $b^{*}$ positivos debido a la presencia de color amarillo. La Fig. 4 muestra las regresiones lineales significativas para los tépalos externos. Las restantes regresiones así como aquellas de los tépalos internos superiores e inferiores no fueron significativas. Se observa correlaciones negativas entre $a^{*} y b^{*}$, $C^{*} \mathrm{yb}^{*}, \mathrm{~h}^{*} \mathrm{y} \mathrm{C}^{*} \mathrm{y}$ correlaciones positivas entre $\mathrm{C}^{*} \mathrm{y} \mathrm{a}^{*}, \mathrm{~h}^{*} \mathrm{y} \mathrm{b}^{*}$.
En todos los casos, los parámetros considerados separan ambos grupos de especies. A medida que $\mathrm{a}^{*}$ aumenta $\mathrm{b}^{*}$ disminuye, lo que implica que en $A$. magnifica hay una combinación de colores azul y rojo mayor que la que presenta $A$. pulchra (Fig 4A) al tiempo que disminuye la saturación (Fig. 4C), dando un aspecto más pálido a $A$. pulchra en comparación con $A$. magnifica. A su vez, la saturación del color rojo es mayor en $A$. magnifica que en A. pulchra (Fig. 4B). 
Gayana Bot. 72(1), 2015

Tabla II. Medidas colorimétricas de los tépalos externos, internos superiores e interno inferior de Alstroemeria magnifica, A. pulchra var. pulchra y A. pulchra var. maxima.

TABLE II. Colorimetric measurements of the outer tepals, upper inner tepals and lower inner tepal of Alstroemeria magnifica, A. pulchra var. pulchra and A. pulchra var. maxima.

\begin{tabular}{|c|c|c|c|c|c|c|}
\hline TAXÓN & $\mathrm{N}^{\circ}$ RECOLECCIÓN & $\mathrm{L}^{*}$ & $a^{*}$ & $b^{*}$ & $\mathrm{C}^{*}$ & $\mathrm{~h}$ \\
\hline \multicolumn{7}{|l|}{ TÉPALOS EXTERNOS } \\
\hline A. magnifica & 4379 & 45,74 & 9,32 & $-11,08$ & 14,47 & $-0,87$ \\
\hline A. magnifica & 4380 & 48,19 & 10,92 & $-10,42$ & 15,09 & $-0,76$ \\
\hline A. magnifica & 4383 & 48,87 & 16,50 & $-12,76$ & 20,85 & $-0,65$ \\
\hline A. magnifica & 4381 & 33,92 & 13,80 & $-10,41$ & 17,28 & $-0,64$ \\
\hline A. magnifica & 4375 & 50,66 & 10,27 & $-8,44$ & 13,29 & $-0,68$ \\
\hline A. pulchra var. maxima & 4385 & 56,85 & 2,81 & 0,93 & 2,95 & 0,31 \\
\hline A. pulchra var. maxima & 4382 & 55,89 & 6,78 & 1,75 & 7,00 & 0,25 \\
\hline A. pulchra var. pulchra & 4384 & 52,09 & 2,90 & $-0,85$ & 3,02 & $-0,28$ \\
\hline \multicolumn{7}{|c|}{ TÉPALOS INTERNOS SUPERIORES } \\
\hline A magnifica & 4379 & 40,92 & 11,66 & 16,51 & 20,21 & 0,95 \\
\hline A. magnifica & 4380 & 42,87 & 8,65 & 21,07 & 22,77 & 1,18 \\
\hline A. magnifica & 4383 & 37,52 & 9,36 & 30,35 & 31,76 & 1,27 \\
\hline A. magnifica & 4381 & 43,03 & 7,18 & 20,44 & 21,66 & 1,23 \\
\hline A. magnifica & 4375 & 48,44 & 4,60 & 28,77 & 29,13 & 1,41 \\
\hline A. pulchra var. maxima & 4385 & 55,81 & 5,81 & 6,75 & 8,90 & 0,86 \\
\hline A. pulchra var. maxima & 4382 & 40,26 & 7,38 & 31,83 & 32,67 & 1,34 \\
\hline A. pulchra var. pulchra & 4384 & 42,98 & 7,07 & 14,33 & 15,97 & 1,11 \\
\hline \multicolumn{7}{|c|}{ TÉPALOS INTERNOS INFERIORES } \\
\hline A magnifica & 4379 & 45,30 & 10,99 & $-12,37$ & 16,54 & $-0,84$ \\
\hline A magnifica & 4380 & 52,52 & 11,34 & $-9,57$ & 14,83 & $-0,70$ \\
\hline A magnifica & 4383 & 47,62 & 14,64 & $-6,60$ & 16,05 & $-0,42$ \\
\hline A magnifica & 4381 & 26,82 & 17,21 & $-8,28$ & 19,09 & $-0,44$ \\
\hline A. magnifica & 4375 & 57,64 & 9,26 & $-8,06$ & 12,27 & $-0,71$ \\
\hline A. pulchra var. maxima & 4385 & 56,20 & 6,60 & $-4,25$ & 7,85 & $-0,57$ \\
\hline A. pulchra var. maxima & 4382 & 42,23 & 10,82 & 0,54 & 10,83 & 0,04 \\
\hline A. pulchra var. pulchra & 4384 & 51,89 & 3,24 & $-0,93$ & 3,37 & $-0,27$ \\
\hline
\end{tabular}

TÉPALOS EXTERNOS

Los espectros de los tépalos externos muestran dos máximos de reflectancia, uno entre los 440 y $480 \mathrm{~nm}$ (azul-violeta) y otro entre 620 y $700 \mathrm{~nm}$ (rojo) (Fig. 4). En A. magnifica los tépalos externos presentaron valores de $\mathrm{a}^{*}$ que fluctúan entre 9,32 y 16,50 mientras en $A$. pulchra a* fluctuó entre 2,81 y 6,78 . Para A. magnifica b* fluctuó entre $-12,76$ y $-8,44$ mientras en $A$. pulchra varió de $-0,85$ a 1,75 (Tabla II). Los valores mayores de $\mathrm{a}^{*} \mathrm{y}$ menores de $\mathrm{b}^{*}$ presentados por $A$. magnifica se manifiestan en el color violáceo más intenso de A. magnifica en comparación con A. pulchra de color más blanquecino. Los valores de croma $\mathrm{C}^{*}$ son también mayores en $A$. magnifica $(13,29-20,85)$ que en $A$. pulchra $(2,95-7,00)$ mientras la luminosidad L* es menor en $A$. magnifica $(33,92-$ $50,66)$ que en $A$. pulchra $(52,09-56,85)$, por lo que los tépalos de $A$. pulchra aparecen más cercanos al blanco en comparación con los de $A$. magnifica. En el espacio CIELab determinado por los parámetros a* y b*, A. pulchra var. maxima (poblaciones 4382 y 4385 ) presentó valores más cercanos a $A$. pulchra var. pulchra (4384) que a las poblaciones de A. magnifica (Fig. 2A, 3A). Las poblaciones de $A$. pulchra se distribuyen en el extremo positivo del eje $b^{*}$ y negativo del eje $a^{*}$, mientras A. magnifica lo hace hacia el extremo positivo del eje $\mathrm{a}^{*} \mathrm{y}$ negativo del eje $\mathrm{b}^{*}$. Ello se debe a la mayor concentración de pigmentos rojos y azules de $A$. magnifica en comparación con $A$. pulchra que contiene mayor cantidad de pigmentos amarillos y verdes. La mayores diferencias totales $(\Delta \mathrm{E})$ en el color de los tépalos externos ocurren entre $A$. magnifica y $A$. pulchra var. pulchra $(17,04)$ y las menores diferencias ocurren entre A. pulchra var. maxima y A. pulchra var. pulchra $(4,51)$ (Tabla III). Las diferencias entre $A$. magnifica y $A$. pulchra var. maxima se deben principalmente al parámetro $\mathrm{b}^{*}$, indicando que los tépalos externos de $A$. magnifica son comparativamente más azules que los de $A$. pulchra var. maxima y al parámetro a* que sugiere que $A$. magnifica presenta tépalos externos relativamente más rojos que $A$. pulchra var. maxima. Ambas especies se separan significativamente a lo largo de ambos ejes (Fig. 2 B-C). 

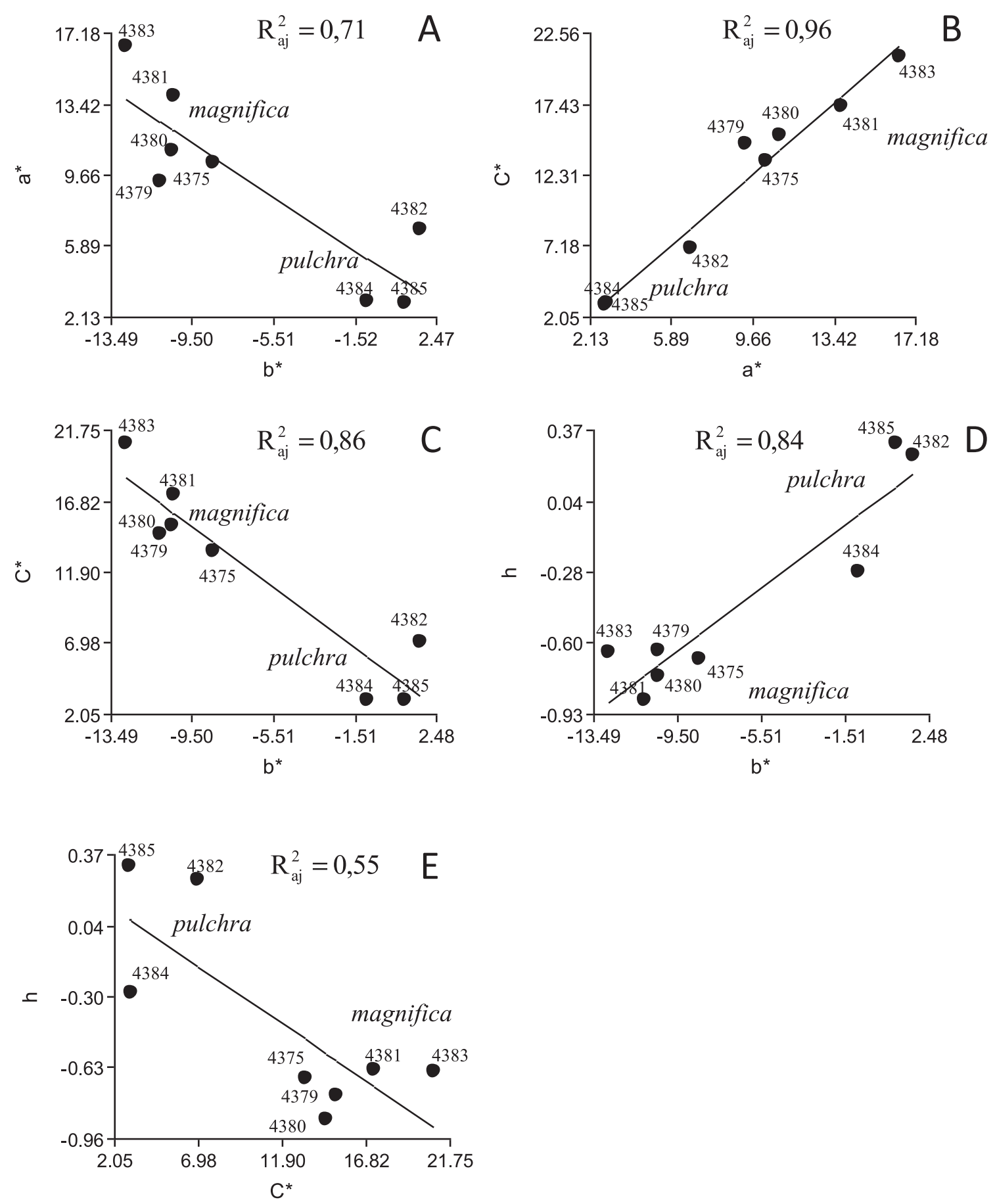

Figura 3. Análisis de regresión lineal entre los parámetros colorimétricos de los tépalos externos para las 8 poblaciones estudiadas. A. a* vs. b*; B. Croma vs. a*; C. Croma vs. b*; D. Hue vs. b*; E. Hue vs. Croma.

Figure 3. Lineal regression analysis among the colorimetric parameters of the outer tepals for the eight studied populations. A. $a^{*}$ vs. $b^{*} ;$ B. Croma vs. a*; C. Croma vs. b*; D. Hue vs. b*; E. Hue vs. Croma.

TÉPALOS INTERNOS SUPERIORES

Con excepción de $A$. pulchra var. maxima que presenta un máximo de reflectancia a nivel de los $440 \mathrm{~nm}$ (azul), los restantes taxones presentan máximos de reflectancia por sobre los $520 \mathrm{~nm}$, indicando la presencia de pigmentos amarillo a rojo (520 a $700 \mathrm{~nm}$ ) (Fig. 4B). Los valores de a* son similares en ambas especies: en $A$. magnifica varió de 4,6 a 11,66 y en $A$. pulchra de 5,81 a 7,38. Los valores de $b^{*}$ fueron, en general, 


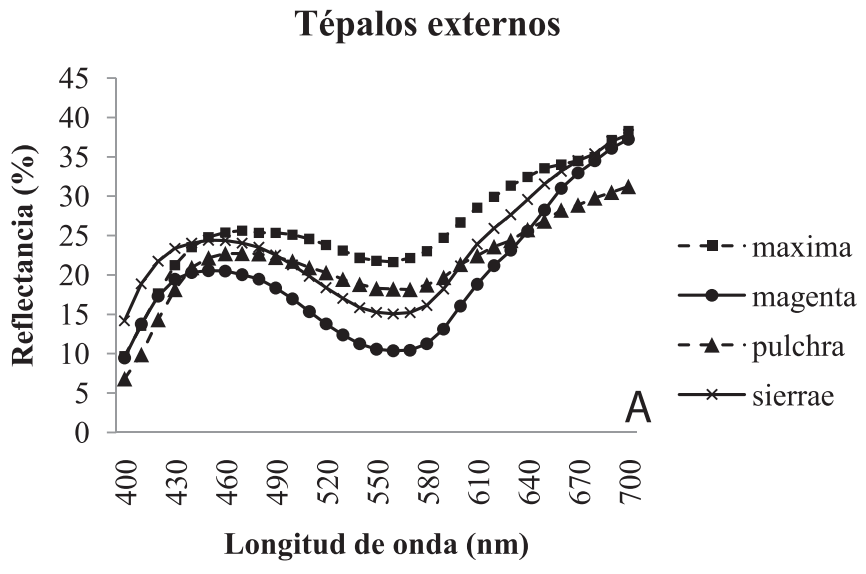

Tépalos internos superiores

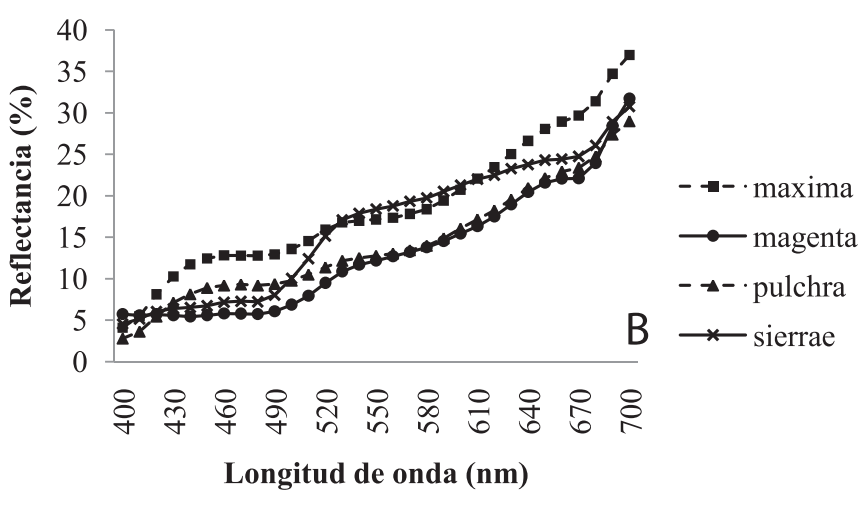

Tépalo interno inferior

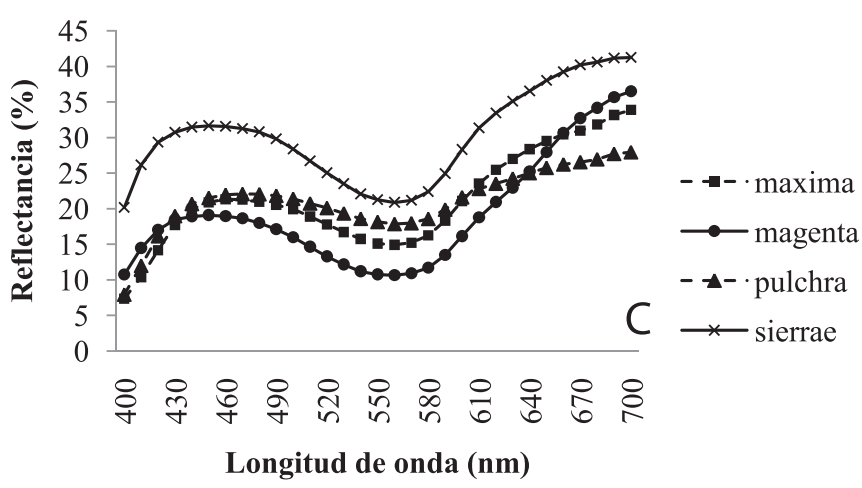

Figura 4. Espectros de reflectancia de los tépalos externos, internos superiores e interno inferior de Alstroemeria magnifica, A. pulchra var. pulchra y A. pulchra var. maxima.

FIGURE 4. Reflectance spectra of the outer tepals, upper inner tepals and lower inner tepal of Alstroemeria magnifica, A. pulchra var. pulchra y A. pulchra var. maxima. más altos que los de los tépalos externos e internos inferiores; para $A$. magnifica b* varió entre 16,51 y 30,35 mientras que para $A$. pulchra, b* varió entre 6,75 y 31,83 . Estos valores más altos se deben a la presencia de pigmentos de color amarillo en los tépalos internos superiores. Las especies no se separan por los parámetros $\mathrm{a}^{*} \mathrm{y} \mathrm{b}^{*}$ de los tépalos internos superiores (gráfico no mostrado). Las diferencias observadas en $a^{*}$ se deben principalmente al color rojo de las manchas púrpuras que presentan los tépalos internos superiores. La población que presentó tépalos con mayor cantidad de manchas púrpuras fue 4379 de Santa Julia y la que presentó menor cantidad de manchas púrpuras fue 4375 de Caleta Hornos. La mayor diferencia total $(\Delta \mathrm{E})$ se observó entre $A$. magnifica y $A$. pulchra (9.19) y la menor diferencia entre $A$. magnifica y $A$. pulchra var. maxima $(7,07)$ (Tabla III).

\section{TÉPALO INTERNO INFERIOR}

Los espectros de reflectancia (Fig. 4) del tépalo interno inferior siguen un patrón comparable al de los tépalos externos. Los tépalos internos inferiores de las flores analizadas presentaron para A. magnifica valores de a* que fluctuaron entre 9,26 y 17,21. Los valores relativamente altos indican color rojo por la mancha rojiza que presentan estos tépalos en la base. Alstroemeria pulchra presentó valores similares para a* $(3,24$ a 10,82 ), indicando menor cantidad de color rojo que en $A$. magnifica. No obstante, para $\mathrm{b}^{*}$ ambas especies mostraron valores diferentes. En A. magnifica b* varió de -12,37 a -6,60 mientras en $A$. pulchra b* varió entre $-4,25$ y 0,54 , indicando que $A$. pulchra se distingue por sus tépalos más blanquecinos (menos azules) que $A$. magnifica. En el espacio CIELab las especies se separan en dos grupos principalmente a lo largo del eje b* (Fig. 2F). La mayor diferencia total $(\Delta \mathrm{E})$ en el color de los tépalos inferiores se observó entre A. magnifica y $A$. pulchra $(29,03)$ y la menor diferencia entre $A$. pulchra var. pulchra y $A$. pulchra var. maxima $(6,16)$ (Tabla III).

\section{ANÁLISIS ESTADÍSTICO}

El análisis de componentes principales (Fig. 6A) separa, a lo largo del primer componente, las especies $A$. pulchra y $A$. magnifica. Hacia el extremo negativo del primer componente se sitúan $A$. pulchra var. pulchra y A. pulchra var. maxima mientras que $A$. magnifica se sitúa en el extremo positivo del eje. El análisis extrajo cinco componentes que explican en conjunto un $99 \%$ de la variabilidad de los datos originales. Los dos primeros componentes explican un 75,5\% de la variabilidad de la matriz. Las variables con mayor aporte al primer componente son $C^{*} \mathrm{e}(0,35), \mathrm{a}^{*} \mathrm{e}(0,34), \mathrm{b} * \mathrm{e}(-0,33)$, $\mathrm{C} * \mathrm{ii}(0,33), \mathrm{a} * \mathrm{ii}(0,31)$ he $(-0,29)$ y $\mathrm{L} * \mathrm{e}(-0,27)$. Las variables con autovectores más altos para el segundo componente son hii $(0,45), b^{*}$ is $(0,41)$, his $(0,41), C *$ is $(0,39)$ y bii $(0,36)$ (Tabla IV). 

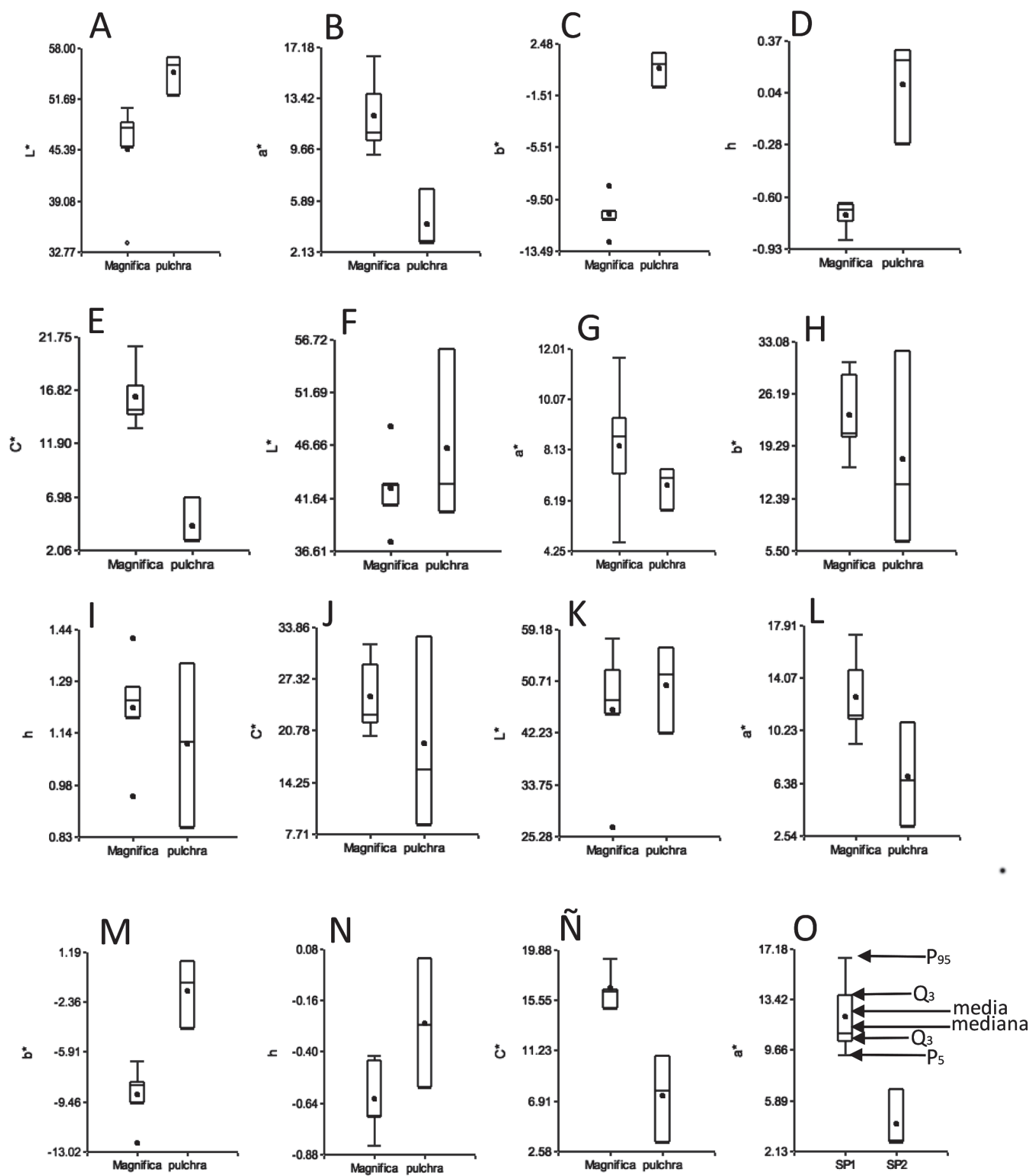

Figura 5. Variabilidad de los parámetros colorimétricos en Alstroemeria pulchra y A. magnifica. Punto = media; Caja $=$ rango intercuartílico (P25-P75).

FiguRE 5. Variability of colorimetric parameters in Alstroemeria pulchra and A. magnifica. Point = mean value; Box = interquartile range (P25-P75). 
Gayana Bot. 72(1), 2015

TABLA III. Diferencias colorimétricas en los parámetros $\mathrm{L}^{*}, \mathrm{a}^{*}, \mathrm{~b}^{*} \mathrm{y}$ diferencia total $\left(\Delta \mathrm{E}^{*}\right)$ entre los taxones estudiados.

TABLE III. Colorimetric differences in parameters $L^{*}, a^{*}, b^{*}$, and total color difference $\left(\Delta E^{*}\right)$ among the studied taxa.

\begin{tabular}{lcccc} 
& $\Delta \mathrm{L}$ & $\Delta \mathrm{a}^{*}$ & $\Delta \mathrm{b}^{*}$ & $\Delta \mathrm{E}$ \\
\hline TÉPALOS EXTERNOS & & & & 17.04 \\
magnifica/pulchra & -10.41 & 5.38 & -12.37 & 16.77 \\
magnifica/maxima & -8.99 & 9.31 & -10.66 & 4.51 \\
pulchra/maxima & 1.42 & 3.93 & 1.71 & 9.19 \\
TÉPALOS INTERNOS SUPERIORES & & & & 7.07 \\
magnifica/pulchra & -0.42 & 1.22 & 9.10 & 7.10 \\
magnifica/maxima & -5.48 & 1.70 & 4.14 & 13.75 \\
pulchra/maxima & -5.06 & 0.48 & -4.96 & 29.03 \\
TÉPALO INTERNO INFERIOR & & & -8.05 & 6.16 \\
magnifica/pulchra & -5.91 & 9.45 & 7.48 & 0.93 \\
magnifica/maxima & 20.75 & -5.47 & . & \\
pulchra/maxima & 2.68 & pulchra var. maxima ; pulchra $=$ A. pulchra var. pulchra
\end{tabular}

TABLA IV. Vectores propios de las variables utilizadas en el análisis de componentes principales.

TABLE IV. Eigenvectors of the variables used in principal component analysis.

\begin{tabular}{|c|c|c|}
\hline \multirow[t]{2}{*}{ VARIABLES } & \multicolumn{2}{|c|}{ VECTORES PROPIOS } \\
\hline & $\mathrm{PC} 1$ & $\mathrm{PC} 2$ \\
\hline $\mathrm{L} *(\mathrm{e})$ & -0.27 & 0.13 \\
\hline$a *(e)$ & 0.34 & 0.06 \\
\hline$b^{*}(e)$ & -0.33 & 0.17 \\
\hline$C^{*}(\mathrm{e})$ & 0.35 & -0.02 \\
\hline $\mathrm{h}(\mathrm{e})$ & -0.29 & 0.19 \\
\hline $\mathrm{L} *$ (is) & -0.23 & -0.23 \\
\hline $\mathrm{a}^{*}($ is $)$ & 0.18 & -0.14 \\
\hline $\mathrm{b}^{*}$ (is) & 0.19 & 0.41 \\
\hline $\mathrm{C} *$ (is) & 0.21 & 0.39 \\
\hline $\mathrm{h}$ (is) & 0.15 & 0.41 \\
\hline $\mathrm{L} *(\mathrm{ii})$ & -0.19 & -0.10 \\
\hline $\mathrm{a} *(\mathrm{ii})$ & 0.31 & 0.07 \\
\hline b*(ii) & -0.24 & 0.36 \\
\hline $\mathrm{C}^{*}$ (ii) & 0.33 & -0.07 \\
\hline $\mathrm{h}$ (ii) & -0.11 & 0.45 \\
\hline
\end{tabular}

Correlación cofenética $=0.936$

e = tépalos externos; is = tépalos internos superiores; $i \mathrm{i}=$ tépalo interno inferior; rcc $=$ índice de correlación cofenética.

\section{DISCUSIÓN}

En algunos grupos de plantas es posible que los caracteres taxonómicos tradicionalmente utilizados no tengan suficiente valor discriminante para diferenciar especies muy cercanas o variedades dentro de un complejo. En estos casos puede ser de mucha utilidad contar con caracteres que brinden una nueva perspectiva sobre el problema. Se ha demostrado que el uso del color de la corola, medido objetivamente, puede tener valor taxonómico cuando los caracteres tradicionales han resultado poco informativos para distinguir especies crípticas (Abdelaziz et al. 2011).
En Alstroemeria, el color de los tépalos ha sido utilizado frecuentemente para distinguir las especies, tanto en las claves de identificación como en las descripciones de los taxones (Bayer 1987, Muñoz \& Moreira 2003, Hofreiter \& Rodríguez 2006, Baeza \& Ruiz 2011). Así por ejemplo, Bayer (1987) utiliza recurrentemente el "color principal de la flor" en su clave para el género, así como la presencia y color de las máculas de los tépalos internos superiores. No obstante, los estudios colorimétricos de los tépalos de Alstroemeria son muy escasos (Norbaek et al. 1998) y el color descrito hace referencia a la percepción subjetiva del mismo por el ojo humano. Ello probablemente explica por qué no ha habido 


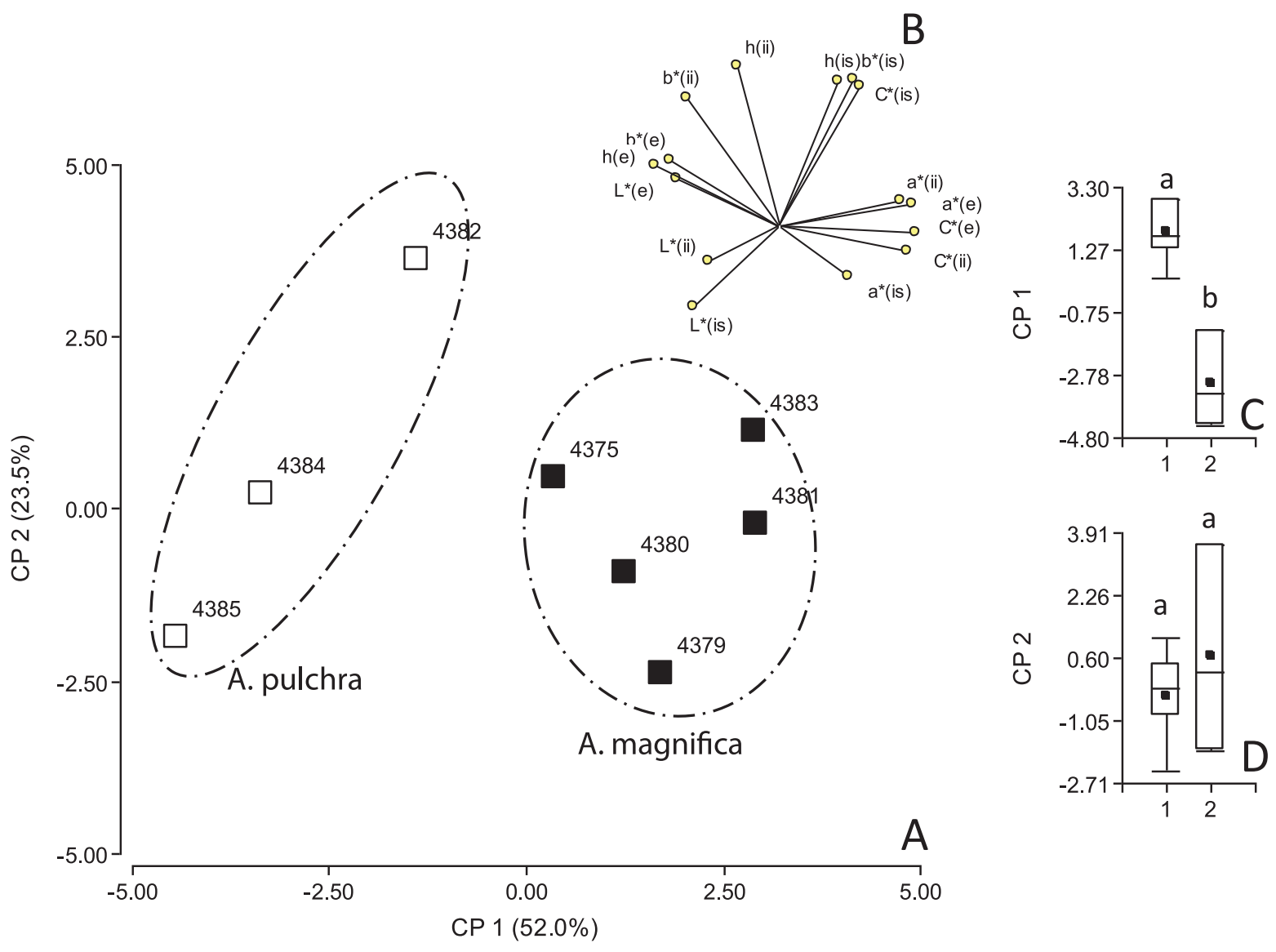

Figura 6. A. Distribución de las OTUs en los dos primeros componentes principales, rec $=0,936$. B. La distribución de las variables se muestra en el extremo superior derecho. C, D. Los gráficos de caja y bigote muestran si la posición de los dos grupos delimitados son o no significativamente diferentes a lo largo de los dos primeros componentes.

FIGURE 6. A. Distribution of the OTUs in the two first principal components; rcc $=0,936$. B. The distribution of the variables is shown on the right corner. C, D. Box-plots show if the position of the delimited groups are or not significatively different along the first and second principal components.

uniformidad de opinión en torno a la posición sistemática de A. pulchra var. maxima.

Por otra parte, es necesario evaluar el color en los distintos tipos de tépalos que presenta la flor, puesto que los tépalos internos suelen ser distintos de los externos y los internos superiores distintos del interno inferior. Así, en el espacio CIELab, A. pulchra var. pulchra y A. pulchra var. maxima se separan de $A$. magnifica por el color de los tépalos externos e interno inferior, pero no por el color de los tépalos internos superiores. Las variables $C^{*}, b^{*}$ y h de los tépalos internos superiores sólo mostraron alta contribución con el segundo componente que separa las poblaciones 4382 y 4385 de A. pulchra var. maxima. Estas poblaciones poseen tépalos internos superiores con coloración algo diferente, especialmente hacia la base (4382 presenta color rojizo más intenso que 4385). Sin embargo, la separación de $A$. pulchra y $A$. magnifica ocurre a lo largo del primer componente, donde las variables más importantes fueron $\mathrm{C}^{*}, \mathrm{a}^{*}, \mathrm{~b}^{*} \mathrm{y} \mathrm{h}$ de tépalos externos. Los tonos más intensos de rojo y azul que presenta $A$. magnifica en comparación con los tonos más pálidos de $A$. pulchra determinados por los parámetros estudiados, coinciden con la percepción humana del color en estas especies. Sin embargo, puesto que el color de la flor es importante para la determinación de las especies, un análisis objetivo de este carácter es deseable. De acuerdo con los resultados obtenidos, sería suficiente con determinar los parámetros $\mathrm{a}^{*}, \mathrm{~b}^{*} \mathrm{y} \mathrm{L} \mathrm{L}^{*}$ en estas especies para distinguirlas, pues ambos parámetros se correlacionan negativamente y de ellos depende el valor de $\mathrm{C}^{*}$. El valor de $\mathrm{L}^{*}$ se correlacionó negativamente con el primer componente, resultando igualmente en un parámetro importante para diferenciar ambas especies.

En A. magnifica los tépalos internos superiores son blancos en la mitad inferior y amarillos en la mitad superior, ambas zonas cubiertas de líneas púrpuras y con una mancha purpúrea más oscura en el ápice sobre fondo lila; las líneas de 
la mitad inferior tienden a juntarse dando un color rojizo a la base del tépalo. En A. pulchra, los tépalos internos superiores siguen un patrón similar de ornamentación y color, aunque las manchas purpúreas forman líneas menos densas y no tienden a juntarse en la base, lo que da un aspecto más claro y luminoso.

El color menos azul de los tépalos externos e interno inferior es el origen del epíteto específico "pulchra". No obstante, hasta ahora sólo se ha considerado la longitud relativa de los tépalos internos superiores con respecto al resto de la flor, notablemente más largos que el resto de los tépalos en $A$. pulchra para distinguirla de $A$. magnifica. En este carácter se basan Muñoz \& Moreira (2003) para transferir la var. maxima de A. magnifica a A. pulchra. En consecuencia, el color de los tépalos externos e interno inferior aparece como un nuevo carácter para distinguir estas especies.

Los resultados de este trabajo soportan la posición sistemática propuesta por Philippi (1864) para A pulchra var. maxima, como lo sostienen posteriormente Muñoz \& Moreira (2003) y no la posición propuesta por Bayer (1987) como variedad de $A$. magnifica. El color de los tépalos externos e interno inferior aparece como un nuevo carácter que junto a la longitud relativa de los tépalos internos superiores y a la anatomía foliar (Lyshede 2002) permite distinguir ambas especies. Este carácter es sorprendente pues ambas especies presentan un color lila de fondo. Si bien es posible captar a ojo desnudo el color más claro y luminoso de $A$. pulchra, en terreno es posible observar variabilidad en el color de las flores en estos taxones. Por ello, el análisis colorimétrico objetivo aparece como un método conveniente y más seguro para captar la diferencia en la tonalidad de la flor que el ojo desnudo. El método es rápido y simple de implementar y su aplicación en taxonomía puede ser de mucha utilidad en éste y otros grupos de plantas. El análisis de color basado en los parámetros CIELab ha sido utilizado en otros grupos de plantas y también en otros órganos de la planta. Así por ejemplo, Fajardo et al. (2008) utilizan datos colorimétricos de las hojas, flores y frutos de Solanum L. en un análisis morfométrico para delimitar las especies de la sección Conicibaccata Bitter. En combinación con caracteres taxonómicos tradicionales, moleculares, palinológicos y anatómicos puede ser una herramienta poderosa. No obstante, los estudios realizados utilizando los parámetros CIELab como caracteres taxonómicos son muy escasos. Los resultados del presente trabajo sugieren que estos caracteres en conjunto con caracteres provenientes de otras fuentes de observación pueden aportar un nuevo enfoque a la delimitación de especies del género Alstroemeria.

\section{AGRADECIMIENTOS}

Trabajo financiado por el Proyecto Fondecyt $\mathrm{N}^{\circ} 1130349$. A María Teresa Valenzuela, Verónica Fuentealba y Cristian Bastías, por la ayuda en terreno y laboratorio. A Alicia Marticorena, curadora del Herbario de la Universidad de Concepción, agradecemos el préstamo del material así como las sugerencias realizadas para mejorar este trabajo. Asimismo, a los correctores anónimos sus valiosas sugerencias.

\section{BIBLIOGRAFÍA}

Aagesen, L. \& A.M. Sanso. 2003. The phylogeny of Alstroemeriaceae, based on morphology, rps16 intron, and rbcL sequence data. Systematic Botany 28: 47-69.

Adbdelaziz, M., J. Lorite, A.J. Muñoz-Pajares, M.B. Herrador, M. Perfectti \&. J.M. Gómez. 2011. Using complementary techniques to distinguish cryptic species: A new Erysimum (Brassicaceae) species from North Africa. American Journal of Botany 98: 1049-1060.

Assis, M.C. 2004. Alstroemeriaceae no Estado do Rio de Janeiro. Rodriguezia 55: 5-15.

BaEzA, C.M. \& E. Ruiz. 2011. Alstroemeria hookeri Lodd. subsp. sansebastiana C.M. Baeza \& E. Ruiz, nueva para la flora de Chile. Gayana Botánica 68: 313-315.

Baeza, C.M., E. Ruiz \& P. NovoA. 2010. The karyotype of Alstroemeria diluta Her. Bayer subsp. chrysantha (Alstroemeriaceae). Chilean Journal of Agricultural Research 70: 667-669.

Bañón, S., A. González, E. Cano, J. Franco \& J. Fernández. 2002. Growth, development and color response of potted Dianthus caryophyllus cv. Mondriaan to paclobutrazol treatment. Scientia Horticulturae 94: 371-377.

BAYer, E. 1987. Die Gattung Alstroemeria in Chile. Mitteilungen der Botanischen Staatssammlung München 24: 1-362.

BuitendiJK, J. \& M. Ramanna. 1996. Giemsa C-banded karyotypes of eigth species of Alstroemeria L. and some of their hybrids. Annals of Botany 78: 449-457.

BuitendiJK, J., E. Boon \& M. Ramanna. 1997. Nuclear DNA content in twelve species of Alstroemeria L. and some of their hybrids. Annals of Botany 79: 343-353.

Chacón, L., A. Sousa, C.M. Baeza \& S.S. Renner. 2012. Ribosomal DNA distribution and a genus-wide phylogeny reveal patterns of chromosomal evolution in Alstroemeria (Alstroemeriaceae). American Journal of Botany 99: 15011512.

Commission Internationale De L'eclairage (CIE). 1978. Recommendations on uniform color spaces: color-difference equations, psychometric color terms. Publ. CIE $\mathrm{N}^{\circ} 15$, Suppl. 2., Paris.

Di Rienzo J.A., F. Casanoves, M.G. Balzarini, L. González, M. Tablada, C.W. Robledo. Infostat Versión 2013. Grupo InfoStat, FCA, Universidad Nacional de Córdoba, Argentina. URL http://www.infostat.com.ar.

Fajardo, D., R. Castillo, A. Salas \& D.M. Spooner. 2008. A morphometric study of species boundaries of the wild potato Solanum series Conicibaccata: A replicated field trial in Andean Peru. Systematic Botany 33(1): 183-192.

Han, T.H., M. De Jeu, H. Van Eck \& E. Jacobsen. 2000. Genetic diversity of Chilean and Brazilian Alstroemeria species assessed by AFLP analysis. Heredity 84: 564-569.

Hatamzadeh, A., A. Razieh, S. Reyhaneh \& B. Davoud. 2012 Comparison of parameters affecting flower color in Gerbera hybrida: A phytochemical study on new varieties. Journal of Agricultural Science 4: 186-194.

Hofreiter, A. \& E.F. Rodríguez. 2006. The Alstroemeriaceae in 
Estudio colorimétrico en Alstroemeria pulchra y A. magnifica. FinOt, V. ET AL.

Peru and neighbouring areas. Revista Peruana de Biología 13: 5-69.

Kashihara, Y., K. Shinoda, N. Murata, H. Araki \& Y. Hoshino. 2011. Evaluation of the horticultural traits of genus Alstroemeria and Bomarea (Alstroemeriaceae). Turkish Journal of Botany 35: $239-245$.

LyshedE, O.B. 2002. Comparative and functional leaf anatomy of selected Alstroemeriaceae of mainly Chilean origin. Botanical Journal of the Linnean Society 140: 261-272.

MuÑoz, M. \& A. Moreira. 2003. Alstroemerias de Chile. Diversidad, distribución y conservación. Consejo Nacional del Libro y la Lectura, Taller La Era, Santiago de Chile, 140 pp.

Norbaek, R., L.P. Christensen, G. Bojesen \& K. Brandt. 1996. Anthocyanins in Chilean species of Alstroemeria. Phytochemistry 42: 97-100.

Norbaek, R., L.P Christensen \& K. Brandt. 1998. An HPLC investigation of flower colour and breeding of anthocyanins in species and hybrids of Alstroemeria. Plant Breeding 117: 63-67.

PhiLIPPI, R.A. 1864. Plantarum novarum chilensium. Linnaea 33: 260-261.

Ruiz, E., K. Balboa, M.A. Negritto, C.M. Baeza, G. Fuentes \& V. BRICEÑo. 2010. Variabilidad genética y morfológica y estructuración poblacional en Alstroemeria hookeri (Alstroemeriaceae) endémica de Chile. Revista Chilena de Historia Natural 83: 605-616.
Rustanius, P., A. Hang, H.G. Hughes \& T. Tsuchiya. 1991. Chromosome analysis of Alstroemeria ligtu hybrids. Hortscience 26: 902-904.

SACKS, E.J. \& D.V. Shaw. 1994. Optimum allocation of objective color measurements for evaluating fresh strawberries. Journal of the American Society for Horticultural Science 119: 330-334.

SaIto. K., M. YamaJI \& T. Honda. 1985: Anthocyanidin glycosides from the flowers of Alstroemeria. Phytochemistry 24: 21252126.

Saito, K., M. Yokoi, M. Ogawa, M. Kamijo \& T. Honda. 1988. 6-Hydroxycyanidin glycoside in the flowers of Alstroemeria. Phytochemistry 27: 1399-1401.

SAnso, A.M. 1996. El género Alstroemeria (Alstroemeriaceae) en Argentina. Darwiniana 34: 349-382.

UphoF, J. C. T. 1952. A review of the genus Alstroemeria. Plant Life 8: $37-53$

Wang, L.S., A. Shiraishi, F. Hashimoto, N. Aoki, K. Shimizu \& Y. SAKATA. 2001. Analysis of petal anthocyanins to investigate flower coloration of Zhongyuan (Chinese) and Daikon Island (Japanese) tree poeony cultivars. Journal of Plant Research 114: 33-43.

Wang, L.S., F. Hashimoto, A. Shiraishi, N. Aoki, J.J. Li \& Y. Sakata. 2004. Chemical taxonomy of the Xibei tree peony from China by floral pigmentation. Journal of Plant Research 117: 47-55.

Recibido: 10.01 .14

Aceptado: 07.01.15 